\title{
Feldforschung im postsowjetischen Raum. Methodologische Reflexion international vergleichender sonderpädagogischer Forschung
}

\begin{abstract}
Zusammenfassung
Die bisherige Forschung zu Exklusionsgefahren für Menschen mit Behinderung und den Handlungsspielräumen nicht staatlicher Akteure in Kasachstan und Usbekistan hat gezeigt, dass empirische Wissenschaft im zentralasiatischen Raum unter herausfordernden Bedingungen stattfindet. Die im folgenden Beitrag anhand einer qualitativen Untersuchung beschriebenen Problemfelder legen eine erhöhte Notwendigkeit der permanenten (Selbst-)Reflexion im Forschungsprozess nahe, die sich jedoch nicht nur auf internationale Kontexte beschränkt.
\end{abstract}

Schlüsselworte: international vergleichende Forschung, empirische Bildungsforschung, Methodologie, Zentralasien

\begin{abstract}
Our research on dangers of excluding people with disabilities and the scope of actions that aim at supporting their inclusion in Kazakhstan and Uzbekistan has revealed the challenging circumstances empirical studies face in intercultural fields. These challenges, as identified in our study, suggest an increased necessity of iterative (self-)reflection in the research process. This is a valuable outcome of our study that is not constraint to international contexts.
\end{abstract}

Keywords: International Comparative Research, Empirical Educational Research, Methodology, Central Asia

\section{Einleitung}

Zentralasien gilt in vielen Bereichen noch immer weitestgehend als terra incognita (Sapper et al., 2007, S. 7). Was für die Region insgesamt gilt, besitzt eine noch stärkere Evidenz für die (Lebens-)Situation von Menschen mit Behinderung vor Ort. Während aktuell immerhin einige englischsprachige Publikationen zu verzeichnen sind (vgl. Rouse \& Lapham, 2013), finden sich in den deutschsprachigen Sammelbänden zur internationalen und vergleichenden Sonderpädagogik keinerlei Be- züge zu Zentralasien (Albrecht et al., 2006; Bürli et al., 2009; Sehrbrock et al., 2013). Im Kontrast zu diesem fehlenden Interesse der deutschen Heil- und Sonderpädagogik eignet sich der zentralasiatische Raum gerade für Fragen der behindertenpolitischen Entwicklungszusammenarbeit, weil durch das einflussreiche Erbe der medizinisch geprägten sowjetischen Defektologie aktuelle Herausforderungen wie inklusive Bildung, De-Institutionalisierung sowie die Entwicklung eines Verständnisses für die sozialen Ursachen von Behinderung besonders groß sind (Kehl, 2018b). Über den behindertenpolitischen Rahmen hinaus lassen sich an dieser Region Spezifika des Forschungsprozesses in interkulturellen Kontexten verdeutlichen, insbesondere die eminent wichtige Rolle der forschenden Person.

\section{Usbekistan und Kasachstan: gesellschafts- politischer und kultureller Kontext}

Usbekistan und Kasachstan erlangten ihre nationalstaatliche Unabhängigkeit mit Auflösung der Sowjetunion vor 30 Jahren. Wie beinahe im gesamten postsowjetischen Raum waren die ersten Jahre der Unabhängigkeit von wirtschaftlichem Niedergang und enormen sozialen Verwerfungen geprägt (Turganbayev, 2013, S. 16f). In den 2000er-Jahren setzte in Kasachstan ein von steigenden Öl- und Gaspreisen getragener Wirtschaftsaufschwung ein, während in Usbekistan bereits mit Ende der 1990er Jahre eine wirtschaftliche Erholung zu beobachten war (OECD, 2016, S. 26).

Im Vergleich zu den dramatischen wirtschaftlichen Folgen der Unabhängigkeit vollzogen sich in anderen gesellschaftlichen Bereichen kaum Veränderungen: Die politische Elite blieb in beiden Ländern weitgehend erhalten (Kubieck, 1998, S. 29; Gharabaghi, 1994, S. 108). Dies mag dazu beigetragen haben, dass bisher keine nachhaltige Demokratisierung stattfinden konnte: Sowohl Kasachstan als auch Usbekistan zählen zu den sog. autoritären Staaten (Ziegler, 2015, S. 5). Inwieweit der Tod des langjährigen (und bis dato einzigen) usbekischen Präsidenten Karimov 2016 (Usbekistan) bzw. der Rücktritt des kasachischen Staatsoberhaupts Nasarbayevs 2019 zukünftig zu Veränderungen führen werden, bleibt abzuwarten (Eschment, 2016, S. 13). 
Beide durch den sowjetischen Einfluss säkularisierten Länder haben nach 1991 eine rasche Islamisierung durchlebt, bleiben jedoch ihrer Verfassung nach säkulare Staaten. Der Islam zeigt sich, wenn auch in unterschiedlichem Maße, als kulturell tief verwurzelter Alltags- und Volksislam. Sowohl in Kasachstan als auch in Usbekistan haben enge Familienstrukturen - über die Sowjetzeit hinweg - ihre historische Bedeutung weitestgehend erhalten (Knox \& Yessimova, 2015, S. 310; Schatz, 2004). Beide Länder haben aus der Sowjetzeit ein vergleichsweise umfassendes Versorgungs- und Bildungssystem für Kinder und Jugendliche mit Behinderung übernommen (Kehl, 2018b; UNICEF, 2013, S. 33). Ebenfalls aus der sozialistischen Vergangenheit stammen die sonderpädagogische Wissenschaft, die sogenannte „Defektologie“, die Behinderungen auf pathologische Störungen zurückführte und soziale Ursachen weitestgehend ignorierte, sowie die Praxis separierender Bildungseinrichtungen für Kinder und Jugendliche mit (geistiger) Behinderung (Šek, 2005).

\section{Ziele und Durchführung der Studie sowie theoretischer Hintergrund}

Bei der im Zeitraum von 2014 bis 2017 in zwei zentralasiatischen Ländern (Kasachstan, Usbekistan) durchgeführten Untersuchung handelt es sich um eine leitfadenbasierte Interviewstudie, in der zwei zentrale Zielstellungen verfolgt wurden: (1) Vor dem Hintergrund, dass Menschen mit Behinderung im Entwicklungskontext zahlreichen Exklusionsgefahren ausgesetzt sind (Yeo, 2001), sollten diese fallspezifisch für Kasachstan und Usbekistan analysiert und die diesen Ausgrenzungsprozessen zugrundeliegenden Mechanismen identifiziert werden. (2) Aufgrund der besonderen Relevanz, welche nationalen und internationalen nicht staatlichen Akteuren in autoritären politischen Systemen zukommt (Ziegler, 2015), sollten die positiven Handlungsspielräume für nicht staatliche Akteure herausgestellt werden, um anschließend zu eruieren, wie diese Handlungsspielräume vor dem Hintergrund von Exklusionsgefahren umgesetzt werden können.

Grundlage der Untersuchung bildeten 29 leitfadenbasierte Interviews, die in mehreren Phasen durchgeführt wurden. Die theoretischen Überlegungen zur Auswahl der Stichprobe orientierten sich grundsätzlich am Theoretical Sampling (Berger-Grabner, 2016). Demnach richten sich Anzahl und Auswahl der Interviewteilnehmenden nach dem Stand der Theoriebildung: Werden durch weitere Interviews keine neuen Erkenntnisse generiert, ist der Punkt einer „theoretischen Sättigung" erreicht. In der praktischen Forschung jedoch erwies sich die Vorgehensweise des Snowball-Samplings, also die Gewinnung von Kontakten über Kontakte, als zentrale Methode der Stichprobengenerierung.

Die überwiegende Mehrzahl der Forschungsinterviews wurde in russischer Sprache ohne dolmetschende Person durchgeführt, drei Interviews in Englisch, zwei in Deutsch und zwei weitere in Usbekisch mit einer dem/der Interviewpartner/-in vertrauten Person als Dolmetscher/-in. Die Länge der Gespräche variierte zwischen 20 Minuten und mehreren Stunden. Die Auswertung der Interviews erfolgte mithilfe der strukturierenden Inhaltsanalyse nach Mayring (2015). Dazu wurden in einem ersten Schritt aus der existierenden Forschungs- literatur deduktiv Kategorien gebildet und diese dann aus einer Analyse der existierenden Forschung zu Exklusionsgefahren für Menschen mit Behinderung im Entwicklungskontext extrahiert. Sie umfassen u.a. Fragen der finanziellen Absicherung, medizinischen Versorgung und statistischen Erfassung von Menschen mit Behinderung (Robson, 2013; Eleweke \& Rodda, 2002). In einem zweiten Schritt wurden diese Kategorien am Forschungsmaterial konkretisiert, wodurch induktiv generierte Unterkategorien entstanden.

Die Forschungsarbeit ist insofern als international vergleichend zu verstehen, als dass sie untersucht, welche Gemeinsamkeiten in den Exklusionsgefahren sowie den Handlungsspielräumen als Tertia Comparationis zwischen diesen beiden Staaten bestehen (Bürli, 1997). Exklusion ist dabei aus soziologischer Perspektive als nicht legitime soziale Schließung auf unterschiedlichen Ebenen zu verstehen (Kronauer, 2013). Dies umfasst Schließungen physischer Art (z. B. durch Zugangsbarrieren), den formellen oder informellen Ausschluss von sozialen, kulturellen und ökonomischen Ressourcen sowie die Verweigerung von Hilfen (Kronauer, 2013, S. 20). Im Sinne funktionaler Äquivalenz international vergleichender Forschung ist Exklusion damit als das komplexe Beziehungsgefüge illegitimer sozialer Schließungen $\mathrm{zu}$ verstehen (Herwartz-Emden, 1995).

Gleichzeitig besitzt die Untersuchung eine interkulturelle Komponente, da sie sich die „Deutungsfolie der kulturellen Spezifität und Differenz" (Allemann-Ghionda, 2004, S. 73) zu eigen macht, wenn es um die Betrachtung von „Behinderung" geht. Denn international vergleichende sonderpädagogische Forschung ist stets mit einer doppelten Andersheit konfrontiert - der Andersheit der Person mit Behinderung ebenso wie der potenziellen Andersheit eines abweichenden kulturellen Kontextes (Erdélyi, 2012). Die Reflexion der eigenen Person als „vergleichendes methodologisches Kriterium“ (Amaral, 2015) gewinnt damit, wie anschließend aufgezeigt wird, besondere Bedeutung. Dieser kulturspezifische Blick auf Behinderung zeigt sich darüber hinaus auch bei der Definition der Handlungsspielräume nichtstaatlicher Akteure, da sie für ihre Legitimation gegenüber der Bevölkerung und staatlichen Akteuren einer spezifisch kulturell geprägten Auffassung von Behinderung folgen müssen.

\section{Herausforderungen im Forschungsprozess}

Die Durchführung von Feldforschung in Zentralasien unterliegt vielfachen Besonderheiten, die eine klare Orientierung an einer distinkten Forschungsmethode enorm erschweren. Haltermann (2013, S. 166) bezeichnet deshalb die Forschung in interkulturellen Kontexten stets als „Hybrid aus Improvisation und Methode“. Die methodischen Herausforderungen lassen sich grob in zwei Schwerpunkte unterteilen. Dies sind zum einen die aus den politisch-kulturellen Bedingungen resultierenden Erschwernisse (1) und zum zweiten die Schwierigkeiten beim Verstehen eines anderen kulturellen Kontextes (2). Beides hat Auswirkungen auf die Durchführung der Untersuchung und die Interpretation der Daten.

(1) Die sicher gravierendste Erschwernis bei der Forschung im zentralasiatischen Raum resultiert daraus, dass die Frage nach der Inklusion immer eine unmittelbare politische 
Dimension beinhaltet (Lorenzkowski, 2006), denn Fragen zur Situation von Menschen mit Behinderung berühren soziale, medizinische und infrastrukturelle Fragen, wie Vertreter nicht staatlicher Strukturen beklagen: „Im Allgemeinen interessiert uns das Leben von Menschen mit Behinderung, aber ihre Probleme stoßen auf die Politik, selbst wenn sie darüber nicht sprechen, führt alles zur Politik“ (Interview XVII ${ }^{1}$, 61-63). Gleichzeitig aber ist eine vollständige Gewährleistung der Anonymität kaum zu realisieren, weil solche Interviews in Usbekistan einer offiziellen Genehmigung bedürfen. Insbesondere die Interviews mit nichtstaatlichen Akteuren, die über staatliche Gängelung und Schikane in ihrer täglichen Arbeit berichteten (siehe Interview XV), untermauern diese Brisanz. Hinzu kommt eine regionsspezifische „Problematik der Imagewahrung“ (Leontiy, 2013, S. 131), die jegliche Kritik am eigenen Land gegenüber Außenstehenden gewissermaßen als „Nestbeschmutzung" versteht. Auf diese Weise kommt in den Interviews neben einer drohenden offiziellen immer auch die Gefahr einer Selbstzensur zum Tragen (Katsui, 2005). Dies erschwert die Interpretation der erhobenen Daten: Wenn positive Entwicklungen in der Behindertenpolitik herausgestellt werden, lässt sich kaum beurteilen, ob dies zwecks „Imagewahrung“ des Landes oder aus Angst vor negativen Konsequenzen geschieht oder aber eine Realität beschreibt. Dies wurde in den Interviews vor allem durch die Diskrepanz zwischen Äußerungen von Betroffenen (Eltern, Menschen mit Behinderung, Vertretenden nichtstaatlicher Strukturen) und Aussagen staatlich institutionalisierter Experten (z. B. Professorinnen im Bereich der Behindertenpädagogik) deutlich. Diese Problematik verdeutlicht auch ein Interview zur Situation von Behindertenorganisationen: "If you come from this approach, blaming the government side - that doesn't work. Because most of the disability groups, they don't want to hear to talk about something blaming the government. Secondly, they will feel concerned to speak something bad about the government (Interview XV, 106-112)." Als erschwerend für den Forschungsprozess erweist sich die immense Bedeutung sozialen Kapitals, verstanden hier „als Nutzung eines dauerhaften Netzes von mehr oder weniger institutionalisierten Beziehungen gegenseitigen Kennens und Anerkennens" (Leontiy, 2013, S. 124), die sich im zentralasiatischen Raum durch das Gewicht von Klan- und Familienstrukturen nochmals verstärkt (Schatz, 2004). Da man für die Rekrutierung von Interviewpartner/-innen auf die Vermittlung innerhalb beruflicher Netzwerke nichtstaatlicher Akteure sowie auf familiäre oder informelle Netzwerke angewiesen ist, kann eine konsequente Orientierung an einer theoretisch begründeten Stichprobenauswahl (Theoretical Sampling) kaum aufrechterhalten werden, wodurch die Stichprobengenerierung stärker durch das Snowball-Sampling geprägt ist (Przyborski \& Wohlrab-Sahr, 2014, S. 184). Unweigerlich bewegen sich Forschende damit in einem bestimmten Netzwerk, in dem tendenziell eher ähnliche Ansichten vertreten werden. Dies verstärkt sich im zentralasiatischen Raum dadurch, dass Netzwerke in der Regel ethnische Zugehörigkeiten sowie eine gemeinsame Sprache teilen und somit häufig übereinstimmende, spezifische kulturelle Werte und Überzeugungen aufweisen. In Usbekistan beispielsweise zeigte sich, dass sich Angehörige einer ethnischen Minderheit (i. d. F. Tadschiken) eher kritisch gegenüber den kulturellen (Rollen-)Vorstellungen im Land äußern, als Ange- hörige der Titularnation: „[S] ie [eine junge Frau, S.K.] verwandelt sich in eine Braut und dann verwandelt sie sich einfach in eine Sklavin. Nicht in allen Familien. [Aber] oft passiert das so. Der Status dieser jungen Frau besteht darin, dass sie sich die ganze Zeit um alle Familienangehörigen kümmern muss, angefangen beim Vater des Mannes, dann seine Mutter und alle weiteren Mitglieder. Sie muss immer am Herd stehen, das Mittagessen zubereiten, das Haus aufräumen und sogar waschen (Interview V, 73-80).“

(2) Eine zweite komplexe Herausforderung, die die Forschung in interkulturellen Kontexten kennzeichnet, betrifft das Verstehen dieses anderen kulturellen Kontextes. Verstehen vollzieht sich immer vor dem Hintergrund der eigenen Sozialisation bzw. des „eigenen Relevanzsystems“ (Mijič, 2013, S. 105). Eine anderer kultureller Kontext stellt für den Außenstehenden somit eine sekundäre Wirklichkeit dar, für deren Repräsentation es ein Wahrheitskriterium im Sinne primärer Wirklichkeit prinzipiell nicht gibt (Kokemohr, 2008, S. 323). Das Verstehen eines anderen kulturellen Kontextes ist unmittelbar mit dem Beherrschen der jeweiligen Sprache verknüpft, weil sich darin die primäre Wirklichkeit widerspiegelt. Zwar verweist auch eine gemeinsame Sprache nicht zwangsläufig auf den gleichen Erfahrungs- und Verständigungshorizont (Mijič, 2013), doch unterscheidet sich in der interkulturellen Forschung nicht nur der (kontextuelle) Sinn eines Wortes, sondern auch dessen (gesellschaftliche) Bedeutung. Wenn Personen mit Behinderung im russischsprachigen Raum vornehmlich als „Behinderte“ (invalid) bezeichnet werden, sind in der deutschen Sonder- und Heilpädagogik andere Personengruppen mit diesem Begriff assoziiert. Dies resultiert auch aus der sowjetischen Tradition der Defektologie und betrifft u.a. die Gruppe von Kindern mit Problemen hinsichtlich ihrer sozioemotionalen Entwicklung, die im sonderpädagogischen Diskurs Zentralasiens kaum auftauchen: „Ungeachtet dessen, dass es diese Kategorie immer gab, haben wir sie den Psychologen überlassen, d. h. die Defektologie hat sie den Psychologen überlassen " (Interview XXIII, 246-47). Das komplexe Erfassen eines anderen kulturellen Kontextes reicht jedoch über das rein sprachliche Verstehen hinaus. Enzenhofer und Resch (2013, S. 210) betonen neben der sprachlichen auch die Notwendigkeit einer Kultur-, Text-, Transfer- und Fachkompetenz. Fachkompetenz umfasst i. d. F. Wissen über die verschiedenen Formen von Behinderung und deren jeweilige (sprachliche und kulturelle) Entsprechung. So ist es zum Verständnis beispielsweise des Begriffs „Lernbehinderung" notwendig, ihn im Sinne funktionaler Äquivalenzen in das jeweilige begriffliche Beziehungsgefüge einzuordnen. Dabei besitzt die direkte englischsprachige Übersetzung (learning disability) kaum eine Entsprechung im Deutschen (Kenneth \& Forness, 2000). Das russischsprachige Äquivalent der zeitweisen Verzögerung der psychischen Entwicklung (vremennaja zaderžka psichičeskogo razvitija) wiederum umfasst auch Kinder mit durchschnittlicher Intelligenzentwicklung, die im deutschsprachigen Raum nicht unter den Begriff der Lernbehinderung fallen (Novikov et al., 1988). Auch deckt sich der deutschsprachige Begriff der geistigen Behinderung nur teilweise mit ähnlichen Begriffen in den beiden anderen Sprachen, was aus der nur hierzulande üblichen Trennung zwischen Lernbehinderung und geistiger Behinderung resultiert (s. Abb. 1). 


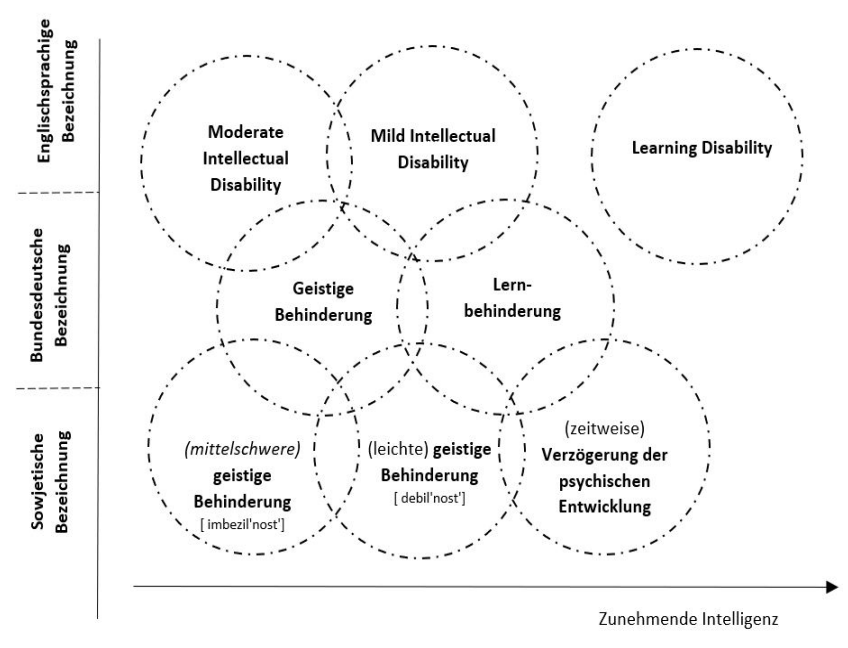

Abb. 1: Beziehungsgefüge verschiedener Begriffstraditionen im deutsch-, englisch-und russischsprachigen Raum im Kontext kognitiver Beeinträchtigung (Quelle: basierend auf Kehl, 2018b, S. 65)

\section{Zum Stellenwert einer permanenten Reflexi- on der eigenen Position (als Forschende)}

In engem Zusammenhang mit den bereits beschriebenen forschungsmethodischen Problemen steht die intersubjektive Nachvollziehbarkeit des Forschungsprozesses. Aus guten Gründen gilt sie als zentrales Gütekriterium der qualitativen empirischen Forschung (Mayring, 2016). Allerdings lassen sich - zum Schutze der Interviewpartner/-innen - nicht alle Ergebnisse dokumentieren, fließen gleichwohl aber unweigerlich in die Interpretation des gesamten Forschungsmaterials ein. Das schließt auch informelle Gespräche und Diskussionen mit ein, die einen wesentlichen Beitrag zum Forschungsprozess leisten. Somit besteht die größte Herausforderung für die Feldforschung in Kasachstan und Usbekistan in der transparenten Dokumentation des Forschungsprozesses.

Das gerade beschriebene Problem verweist auf die besondere Bedeutung der durch Steinke (1999, S. 231ff.) postulierten „reflektierten Subjektivität“: Wenn die forschende Person selbst explizit als Bestandteil des Forschungsprozesses und ihre Subjektivität als Teil der Methode verstanden wird, müssen die durch den Forschenden eingebrachten subjektiven Anteile, die den Forschungsprozess beeinflusst haben könnten, identifiziert und transparent gemacht werden. Im Kern geht es hier also um die Frage, wie die Rolle des oder der Forschenden als Subjekt und als Teil der untersuchten sozialen Welt methodisch reflektiert wird. Die Auseinandersetzung mit dieser Frage ist dabei auch als Beitrag zu werten, einer Vereinfachung von Wissenschaft, die durch die Vereinfachung ihrer Akteure hervorgebracht wird, zu begegnen (Feyerabend, 2016, S. 15). In Orientierung an den von Steinke formulierten Leitfragen sollen im Folgenden exemplarisch einige Aspekte ausgeführt werden, die an der Untersuchung im zentralasiatischen Raum evident geworden sind.

Steinke (1999, S. 234) betont u.a. die Notwendigkeit der Reflexion biographischer Beziehungen des Forschenden und seiner kulturellen Herkunft, was sich im Rahmen der dargestellten Forschung anhand der Problematik rund um die Stichprobenauswahl untermauern lässt. Der Zugang zu einem bestimmten Netzwerk gelingt möglicherweise gerade da, wo Überzeugungen und Ansichten den eigenen Positionen nahestehen. Wenngleich dieser Aspekt für jeglichen Forschungsprozess gilt, verstärkt er sich im interkulturellen Feld durch die Begrenzungen der sprachlichen Verständigung. So spielten die fließenden Russischkenntnisse des Interviewers zweifelsohne eine wesentliche Rolle bei der Generierung von Interviewpartner/-innen und beim Aufbau einer vertrauensvollen Beziehung, während ihm dies weder in Usbekisch noch Kasachisch möglich gewesen wäre. Nun unterscheidet sich jedoch die Sozialisation einer russischsprachigen Person vor allem in Usbekistan meist deutlich von derjenigen der Titularnation - und damit mitunter auch der Blick auf die Situation von Menschen mit Behinderung. Auch die implizite Hierarchie, die sich aufgrund der kulturspezifischen Rollen von Frau und Mann ergibt, kann den Forschungsprozess beeinflussen. Herausforderungen ergeben sich auch aus dem Verhältnis zwischen Nähe und Distanz im Forschungsprozess. Der Interviewer war, als aus dem westlichen Ausland stammender Forscher, eindeutig Fremder dieses lokalen kulturellen Kontextes, gleichzeitig als vor Ort angestellter Dozent aber fest in einer lokalen Bildungsinstitution verankert. Dieser mehrjährige und permanente Aufenthalt im Feld birgt die Gefahr, dass der Vergleich nicht nur als wissenschaftliche Methode, sondern als „universelle mentale Operation “ durch einen ,involvierten und engagierten Beobachter" vorgenommen wird (Amaral, 2015, S. 124). Insbesondere der akademische Hintergrund des Forschenden als Behindertenpädagoge könnte die Angleichung des Anderen an das Eigene (Nostrifizierung) verstärken, da dieses Hintergrundwissen möglicherweise zu einem oberflächlichen Verstehen, der Situation von Menschen mit Behinderung verleitet.

Die durch den langen Feldaufenthalt entwickelten Beziehungen zu einer Reihe von Gesprächspartner/-innen, die weit über die Dichotomie Forschende/Beforschte hinausgehen, erschwert die Wahrung einer kritischen Distanz im Forschungsprozess zusätzlich. Gleichwohl ist der Aufbau eines stabilen Vertrauensverhältnisses alternativlos. Wie komplex sich die Situation in der konkreten Feldforschung darstellt, zeigt sich daran, dass beispielsweise Schilderungen und Erlebnisse zum Umgang mit Menschen mit Behinderung nicht kritisch hinterfragt werden können, um aufgebautes Vertrauen nicht zu zerstören (Mijič, 2013).

Vor dem Hintergrund der Bedeutung einer bestimmten sozialen und kulturellen Sozialisation für die Wahrnehmung eines anderen kulturellen Kontextes ist die von Steinke (1999, S. 234) geforderte "gleichschwebende Aufmerksamkeit“" gegenüber allen Phänomenen im interkulturellen Forschungsprozess besonders herausfordernd. Aus der Sozialisation im eigenkulturellen Kontext erwächst eine normative Erwartungshaltung gegenüber dem fremdkulturellen Umfeld. So war während der Feldforschung die Unsichtbarkeit behinderter Menschen im gesellschaftlichen Alltag in einem solchen Maße auffällig, dass sich der Blick der forschenden Person stark auf Exklusionsprozesse fokussierte. Exempel für gelungene Inklusion von Menschen mit Behinderung blieben möglicherweise unbemerkt und wichtige Erkenntnisse über vorhandene Ressourcen konnten nicht gewonnen werden. 


\section{Fazit \& Ausblick}

Die aufgezeigten Herausforderungen im Forschungsprozess in Zentralasien legen zunächst eine grundlegende erkenntnistheoretische Bescheidenheit nahe, die sich nicht nur auf das interkulturelle Feld beschränkt, sich hier aber besonders zeigt, da das Verstehen eines fremdkulturellen Kontextes per se erheblichen Einschränkungen unterliegt und die Spezifika des Feldes das strikte Einhalten methodischer Vorgaben erschweren. Eine gewisse Offenheit gegenüber notwendigen Modifikationen methodischer Vorgehensweisen an die Bedingungen, unter denen ein konkreter Forschungsprozess stattfindet, wird so unverzichtbar für den Erkenntnisprozess. Dies allerdings führt zu einer permanenten Gratwanderung zwischen - objektivitätssichernden - Verfahrensregeln und im Prozess notwendig werdenden Abweichungen. Die interkulturelle Forschung erweist sich hier gewissermaßen als Brennglas für die methodologischen und methodischen Probleme qualitativer Forschung. Vermutlich muss sie sich einem gewissen Pragmatismus unterwerfen, der in methodischer Hinsicht jenem ähnelt, den Knorr-Cetina (1991, S. 90) für die Naturwissenschaften als „opportunistische Logik der Forschung" beschreibt. Forschungsergebnisse sind demnach stets das Produkt komplexer Umstände und Aushandlungsprozesse, in diesem Sinne also kontextuell kontingent.

Dem Dilemma, dass zentrale wissenschaftliche Gütekriterien an merkbare Grenzen stoßen, lässt sich nur dadurch begegnen, dass der permanenten (Selbst-)Reflexion ein noch größerer Stellenwert eingeräumt werden muss. Andernfalls potenziert sich in der interkulturellen Feldforschung eine Gefahr, die auch für die empirische Forschung im Allgemeinen gilt: Eine zu starre Orientierung an methodischen Vorgaben führt zu einer oberflächlichen Vereinheitlichung des Untersuchungsgegenstandes. In letzter Konsequenz ordnet sich das zu untersuchende Phänomen damit der Methode unter (Mayring, 2016, S. 149). Diese Kritik ist zwar keinesfalls mit der radikalen Absage Feyerabends (2016) an jedwede methodischen Vorgaben gleichzusetzen, die Gefahr einer Vereinfachung von komplexen Fragestellungen bleibt jedoch zu bedenken. Eine permanente (Selbst-)Reflexion kann dieser Simplifizierung von Forschungsprozessen - und damit auch von Wissenschaft allgemein - entgegenwirken.

\section{Anmerkungen}

1 Die Nummerierung bezieht sich auf den Ursprung der Interviews in Kehl (2018b).

\section{Literatur}

Albrecht, F., Bürli, A. \& Erdélyi, A. (2006). Internationale und vergleichende Heilund Sonderpädagogik: aktuelle Diskussionen, Ergebnisse und Herausforderungen. Bad Heilbrunn: Klinkhardt.

Allemann-Ghionda, C. (2004). Einführung in die Vergleichende Erziehungswissenschaft. Weinheim: Beltz.

Amaral, M.P. (2015). Methodologie und Methode in der International Vergleichenden Erziehungswissenschaft. In M. P. Amaral, \& S. K. Amos, (Hrsg.), Internationale und Vergleichende Erziehungswissenschaft, Geschichte, Theorie, Methode und Forschungsfelder (S. 107-132). Münster: Waxmann.

Berger-Grabner, D. (2016). Wissenschaftliches Arbeiten in den Wirtschafts- und Sozialwissenschaften. Hilfreiche Tipps und praktische Beispiele. (3., aktualisierte und erweiterte Auflage). Wiesbaden: Springer Gabler. http://dx.doi.org/10.1007/978-3658-13078-7.

Bürli, A. (1997). Sonderpädagogik international. Vergleiche, Tendenzen, Perspektiven. Luzern: SZH/SPC.

Bürli, A., Strasser, U. \& Stein, A.-D. (2009). Integration/Inklusion aus internationaler Sicht. Kempten: Julius Klinkhardt.

Dados, N., \& Connell, R. (2012). The global south. Contexts, 11(1), 12-13. http:// doi.org/10.1177/1536504212436479

Eleweke, J. \& Rodda, M. (2002). The challenge of enhancing inclusive education in developing countries. International Journal of Inclusive Education, 6(2), 113-126. Enzenhofer, E. \& Resch, K. (2013). Unsichtbare Übersetzung? Die Bedeutung der Übersetzungsqualität für das Fremdverstehen in der qualitativen Sozialforschung. In R. Bettmann und M. Roslon (Hrsg.), Going the Distance. Impulse für die interkulturelle Qualitative Sozialforschung (S. 203-230). Wiesbaden: Springer.

Erdélyi, A. (2012). Leben mit dem Anderssein im internationalen Kontext - auf der Suche nach methodischen Zugängen. In A. Erdelyi, H.-P. Schmidtke \& P. Sehrbrock (Hrsg.), International vergleichende Heil-und Sonderpädagogik weltweit: Grundlagen, Migration, „Dritte Welt“, Europa (S. 39-56). Bad Heilbrunn: Julius Klinkhardt.

Eschment, B. (2016). Alles wie immer - oder doch anders? Die Präsidentenwahl in Usbekistan und ihre Folgen. In Zentralasienanalysen, 108, 12-13. Zugriff am 10.02.2017. http://www.laender-analysen.de/zentralasien/pdf/ZentralasienAnalyse n108.pdf

Fazendeiro, B. (2015). Keeping the face in the public sphere: recognition, discretion and Uzbekistan's relation with the United States and Germany, 1991-2006. Central Asian Survey, 34(3), 341-356.

Feyerabend, P. (2016). Wider den Methodenzwang. Frankfurt a. M.: Suhrkamp.

Gharabaghi, K. (1994). Development Strategies for Central Asia in the 1990s: In Search of Alternatives. Third World Quarterly, 15(1), 103-119.

Grech, S. \& Soldatic, K. (2016). Disability in the Global South. The Critical Handbook. Springer International Publishing: Cham.

Haltermann, I. (2013). Ich habe meinen Anwalt bei mir ... Der Zugang zum Feld im Kontext interkultureller Forschung. In R. Bettmann und M. Roslon (Hrsg.), Going the Distance. Impulse für die interkulturelle Qualitative Sozialforschung (S. 149-168). Wiesbaden: Springer.

Katsui, H. (2005). Towards Equality. Creation of the Disability Movement in Central Asia. Zugriff am 20.05.2017 Helsinki:https://digitalcommons.ilr.cornell.edu/cgi/ viewcontent.cgi? article $=1333 \&$ context $=$ gladnetcollect

Kehl, S. (2018a). Handlungsspielräume nationaler und internationaler nicht-staatlicher Akteure zur Unterstützung von Menschen mit Behinderung in Kasachstan und Usbekistan. Sonderpädagogische Förderung heute, 63(1), 88-99.

Kehl, S. (2018b). Exklusionsgefahren für Menschen mit Behinderung in Kasachstan und Usbekistan sowie Handlungsspielräume zur Unterstützung ihrer Inklusion durch nationale und internationale nicht-staatliche Akteure. Dissertation. Rostock: Universität. https://doi.org/10.18453/rosdok_id00002333

Kenneth A. K. \& Forness, S. R. (2000). What Definitions of Learning Disability Say and Don't Say. A Critical Analysis. Journal of Learning Disabilities, 33(3), 239256.

Knorr-Cetina, K. (1991). Die Fabrikation von Erkenntnis. Frankfurt a. M.: Suhrkamp.

Knox, C. \& Yessimova, S. (2015). State-Society Relations: NGOs in Kazakhstan. Journal of Civil Society, 11(3), 300-316.

Kokemohr, R. (2008). Kulturelle Prafigurationen sozialer Interaktion. Methodologische Fragen interkultureller Kooperation, diskutiert an einem Beispiel aus Kamerun. In G. Cappai (Hrsg.): Forschen unter Bedingungen kultureller Fremdheit (S. 307-328). Wiesbaden: VS Verlag für Sozialwissenschaften.

Kronauer, M. (2013). Soziologische Anmerkungen zu zwei Debatten über Inklusion und Exklusion. In R. Burtscher, E. J. Ditschek, K.-E. Ackermann, M. Kil, \& M. Kronauer (Hrsg.), Zugänge zur Inklusion. Erwachsenenbildung, Behindertenpädagogik und Soziologie im Dialog (S. 17-26). Bielefeld: Bertelsmann.

Leontiy, H. (2013). Feldforschungszugang als Herausforderung der interkulturellen Fallstudien am Beispiel deutsch-ukrainischer Wirtschaftskommunikation. In R. Bettmann und M. Roslon (Hrsg.), Going the Distance. Impulse für die interkulturelle Qualitative Sozialforschung (S. 199-148). Wiesbaden: Springer.

Lorenzkowski, S. (2006). Professional Inclusion in Uzbekistan. Behinderung und Dritte Welt. Journal for Disability and International Development, 2(2006), 17-22. Zugriff am 14.06.2017. http://www.zbdw.de/projekt01/media/pdf/20062.pdf

Mayring, P. (2015). Qualitative Inhaltsanalyse: Grundlagen und Techniken. Weinheim Beltz: Pädagogik.

Mayring, P. (2016). Einführung in die qualitative Sozialforschung. Eine Anleitung zu qualitativem Denken. Weinheim und Basel: Beltz.

Mijič, A. (2013). Grenzen des Verstehens? - Verstehen der Grenzen! Reflexionen über die methodischen Herausforderungen hermeneutischer Sozialforschung im ,interkulturellen' Kontext. In R. Bettmann \& M. Roslon (Hrsg.), Going the Distance. 
Impulse für die interkulturelle Qualitative Sozialforschung (S. 95-118). Wiesbaden: Springer.

Nohl, A.-M. (2008). Interkulturelle Kommunikation. Verständigung zwischen Milieus in dokumentarischer Interpretation. In G. Cappai (Hrsg.), Forschen unter Bedingungen kultureller Fremdheit (S. 281-305). Wiesbaden: Verlag für Sozialwissenschaften.

Novikov, L., Freiburg, G. \& Jehle, P. (1988), Entwicklungen im Sonderschulwesen der Sowjetunion und der Deutschen Demokratischen Republik. Köln \& Wien: Böhlau.

OECD (2016): Multi-dimensional Review of Kazakhstan. Ausgabe 1. Initial Assessment. Zugriff am 10.10.2016. http://www.keepeek.com/Digital-Asset-Management/oecd/development/multi-dimensional-review-of-kazakhstan_97892642 46768-en\#page1

Przyborski, A. \& Wohlrab-Sahr, M. (2014). Qualitative Sozialforschung. Ein Arbeitsbuch. (4., erw. Aufl.) München: Oldenbourg. http://dx.doi.org/10.1524/97834 86719550 .

Robson, C. (2013). Education Children with Disabilities in Developing Countries: The Role of Data Sets. Zugriff am 10.04.2015 http://siteresources.worldbank.org/DISABILITY/Resources/280658-1172610312075/EducatingChildRobson.pdf

Rouse, M. \& Lapham, K. (2013). Learning to see invisible children. Inclusion of Children with disabilities in Central Asia. Open Society Foundation. Budapest.

Sapper, M., Weichsel, V. \& Huterer, A. (2007). Mosaikstein. In Deutsche Gesellschaft für Osteuropakunde e.V. (Hrsg.): Machtmosaik Zentralasien. Traditionen, Restriktionen, Aspirationen. Osteuropa, 57(8-9), 7.

Schatz, E. (2004). Modern Clan Politics: The Power of „Bloo” in Kazakhstan and Beyond. Jackson School Publications in International Studies: University of Washington Press.

Sehbrock, P., Erdélyi, A. \& Gand, S. (2013). Internationale und vergleichende Heilund Sonderpädagogik und Inklusion: Individualität und Gemeinschaft als Prinzipien internationaler Heil- und Sonderpädagogik. Kempten: Julius Klinkhardt.

Šek, O. (2005). Sozialer Ausschluss von Menschen mit Behinderung in der UdSSR [Social'noe isključenie invalidov v SSSR] In P. Romanova et al. (Hrsg.), Not und Elend. Die Geschichte der sozialen Arbeit in Russland im 20. Jahrhundert [Nužda i beda. Istorija social'noj raboty v Rossii, XX. v.]. Saratov: Nauchnaja Kniga.

Steinke, I. (1999). Kriterien qualitativer Forschung. Ansätze zur Bewertung qualitativ-empirischer Sozialforschung. Weinheim: Juventa.

Turganbayev, Y. (2013). Convergence across Kazakhstan regions. Aberdeen: Robert Gordon University Aberdeen.

UNICEF (2013). Social Protection Priorities in Uzbekistan. Taking Stock and Looking Forward. UNICEF Country Office in Uzbekistan.

Yeo, R. (2001). Chronic Poverty and Disability. Zugriff am 11.06.2017 https://papers. ssrn.com/sol3/Delivery.cfm/SSRNID1754542code1382430.pdf?abstractid=1754 $542 \&$ mirid $=1 \&$ type $=2$

Ziegler, C. H. (2015). Introduction. In C. H. Ziegler (Hrsg.), Civil Society and Politics in Central Asia (S. 1-18). Kentucky: University Press of Kentucky.

\section{Dr. Katja Koch}

ist Professorin für Sonderpädagogik mit dem Förderschwerpunkt geistige Entwicklung an der Universität Rostock. Ihr Weg führte sie nach einem Studium in Rostock für eine Habilitation an die Universität Würzburg, wo sie sich hauptsächlich mit den Themen Soziologie der Behinderung und (frühe) Förderung der kognitiven Entwicklung beschäftigte. Seit 2007 berufen an die Universität Rostock ist sie nach wie vor und mit Freude in der Lehramtsausbildung tätig, wichtige Arbeitsschwerpunkte bilden hier die Themen Didaktik und Inklusion. Zudem beschäftigt sie sich intensiv mit der Pädagogik in der DDR und in postsowjetischen Ländern.

\section{Dr. Stephan Kehl}

ist seit Oktober 2020 an der Pädagogische Hochschule Ludwigsburg im Arbeitsbereich Psychologie und Diagnostik des Förderschwerpunktes geistige Entwicklung tätig. Nach einem mehriährigen Forschungs- und Arbeitsaufenthalt in Ländern der ehemaligen Sowjetunion wurde er über die Handlungsspielräume nicht staatlicher Akteure sowie die Exklusionsgefahren für Menschen mit Behinderung in Usbekistan und Kasachstan promoviert. Anschließend war er an der Universität Rostock tätig. Seine Forschungsschwerpunkte liegen neben der Sonderpädagogik im postsowjetischen Raum auf den Arbeitsgedächtnisbesonderheiten bei Personen mit sogenannter geistiger Behinderung. 